\title{
Projecting future births with fertility differentials reflecting women's educational and migrant characteristics
}

\author{
Michaela Potančoková ${ }^{1,2, *}$ and Guillaume Marois ${ }^{3,1}$
}

\begin{abstract}
Building on the well-established knowledge on fertility differentials by education and nativity/migration status, we employ microsimulation modelling to demonstrate the effect of accounting for such differences in population projections. We consider fertility differentials by educational attainment, enrolment in full-time education, region of birth, age at immigration, and duration of stay in the host country, which we introduce step-wise into the microsimulation model for the EU28. Results on projected TFRs and births by 2060 illustrate the importance of accounting for several sources of population heterogeneity. In the context of future educational expansion, modelling education differentials for students and for women with completed education is needed to capture the postponement effect of education on childbearing. Future migration assumptions that include migrant fertility differentials lead to widely varying projected numbers of future births. At fixed fertility differentials and a fixed composition of immigrant flows, the net effect of immigrant fertility on the overall TFR in the EU28 is projected to increase from the estimated 0.12 in 2015-2019 to 0.17 in 2055-59 in the scenario with baseline migration, and to 0.25 in $2055-59$ in the scenario with doubled migration.
\end{abstract}

Keywords: population projection; microsimulation; fertility; education; immigrants

\footnotetext{
${ }^{1}$ International Institute for Applied Systems Analysis (IIASA), Wittgenstein Centre for Demography and Global Human Capital (IIASA, OeAW, University of Vienna), Laxenburg, Austria

${ }^{2}$ Formerly at the Joint Research Centre - European Commission

${ }^{3}$ Asian Demographic Research Institute (ADRI), Shanghai University, Baoshan, Shanghai, China

${ }^{*}$ Correspondence to: Michaela Potančoková, potancok@iiasa.ac.at
} 


\section{Introduction}

Past research has established that fertility rates among women differ by their level of education (e.g., Rindfuss and Bumpass 1980; Kravdal 2002; Lutz and Skirbekk 2014) and by their country of birth (e.g., Sobotka 2008; Kulu et al. 2019). How important is it to take these differentials into account when projecting the population of the EU? To what extent does using different approaches to modelling fertility affect the projected number of births? Both the expansion of education and the immigration of foreign nationals into the EU are trends that are likely to continue in the future, and that will play a role in shaping the population of the EU.

We argue that the multi-dimensional modelling of fertility is needed to capture the implications of differences in fertility across socio-economic groups, as the EU population is becoming increasingly diverse as a result of higher and more diversified migration flows in many EU countries in recent decades. When projecting population, it is important to account for immigration from third countries with generally higher fertility levels than the host EU countries, because it impacts the composition of the female population of reproductive age (by nativity, and also by education) - and, consequently, the number and the structure of births, as well as the size and the composition of the population. As differences in fertility between the native-born population and various groups of immigrants are partly associated with educational attainment (e.g., Roig Vila and Castro-Martín 2007), it is important to capture both dimensions simultaneously. Building on the well-established knowledge about differentials in fertility by education and migration status, we employ microsimulation modelling to demonstrate the effect of accounting for such differences in population projections. We focus on differences in the projected total fertility rate (TFR) and in the number of births and their composition. We present several scenarios that differ in how they model fertility and in the migration assumptions they use. As this work has been developed within the CEPAM project, ${ }^{1}$ it focuses on EU28 countries.

Conventionally, multistate population projection methods (Rogers 1980, 1986; Schoen 1988) are used to take into account population diversity beyond the standard components of age and sex. The dimensions that are usually included in multistate projections influence population dynamics, and are associated with differences in fertility, mortality, and the propensity to migrate, such as educational attainment, which is closely linked to all components of population change (Lutz and Goujon 2001; Lutz et al. 2014). Among the other dimensions that are commonly included in such projections are place of birth (Rogers et al. 1999), religion (Goujon et al. 2007; Stonawski et al. 2015), ethnicity (Rees 2002; Raymer et al. 2018), and race (Colby and Ortman 2015). Multistate population projections usually include one, or at most two dimensions in addition to age and sex because adding three or more dimensions

\footnotetext{
The Centre for Expertise on Population and Migration (CEPAM) is a joint research project between IIASA and the Joint Research Centre of the European Commission that studies the future changes in EU populations under various alternative migration scenarios.
} 
at the same time increases the complexity of this type of model to the point where it is difficult to handle any additional state. In a full multistate model, each additional state multiplies the set of transitions between the states. Data limitations also play an important role, particularly when the projection is for a group of countries with differing levels of data quality.

In contrast, microsimulation modelling offers a flexible way of taking into account a larger number of characteristics simultaneously. It is easier to handle a large number of parameters, because adding a dimension does not require multiplying all of the others. Adding a dimension simply involves adding a single column in the database. Among the advantages of microsimulation models are that they can provide richer outputs, and they can be used to build individual-level determinants of events. Thus, such models can provide better results when the number of dimensions included is relatively large, and when handling interactions between multiple variables (van Imhoff and Post 1998). The CEPAM-mic microsimulation model captures both the educational and the migrant characteristics of individuals, and its dimensions include age, sex, educational attainment, region of origin, duration of stay in the host country, and several others detailed in section 3.2. Importantly, microsimulation facilitates the appropriate modelling of period fertility because it enables us to include (rather than to adjust for) tempo effects in fertility that arise due to educational enrolment and the interplay between the migration process and childbearing.

This paper is structured as follows. As a backdrop to the modelling of fertility, we summarise the most enduring findings on fertility differentials by the educational and the migrant characteristics of women. In the data and methods section, we explain our general approach of contrasting the conventional multistate method with the microsimulation, while specifically focusing on the approaches to and the challenges involved in modelling fertility differentials. We then introduce the projection scenarios. In the data section, we also describe the CEPAM-mic microsimulation model, including its modules, its baseline population estimates, and its parameters. In a dedicated subsection, we present in detail the fertility module, including the data and estimation procedures for the parameters, and the results obtained using logit regression on Labour Force Survey data. In the results section, we focus on the compositional effects of education and migration on overall TFRs, and the number of births in the EU28 countries estimated under different projection scenarios. The scenarios differ in terms of the fertility modelling approaches and the migration assumptions they use to illustrate the compositional effects, and in the sensitivity of the models to the assumed migration volumes. We conclude with reflections on the relevance and the limitations of our exercise.

\section{Background}

Across social groups, fertility levels, the timing of parenthood, and fertility ideals can vary substantially (e.g., Berrington and Pattaro 2014). Among the 
socio-economic variables that influence fertility, the educational attainment of women stands out as having a strong and consistent negative relationship with fertility (Rindfuss and Bumpass 1980; Jeejebhoy 1995; Kravdal 2002; Bongaarts 2003; Lutz and Skirbekk 2014). Compared to their less educated counterparts, educated women tend to have fewer children later in life, and are more likely to be childless. Educational expansion is a major driver of the postponement of parenthood, because in modern, post-industrial societies, schooling extends well into reproductive ages (Neels et al. 2017; Ní Bhrolcháin and Beaujouan 2012), and family formation is often normatively conditioned on the completion of education (Blossfeld and Huinink 1991; Mortimer et al. 2005). Analyses of series of completed cohort fertility rates have shown that education differentials tend to decline as the overall fertility rate decreases, and that they tend to be more pronounced among older than younger cohorts. Such differentials are smaller in institutional settings that mitigate the high opportunity costs of childbearing with policies that promote work-family balance, gender equity, and affordable childcare (Kravdal and Rindfuss 2008; Wood et al. 2014). Cohort differentials in fertility by education have diminished in some Western and Northern European countries (van Bavel 2014; Jalovaara et al. 2019), but strong gradients persist, particularly in Eastern Europe (Basten et al. 2014; Wood et al. 2014; Brzozowska 2016).

International migration is another important factor underlying the fertility differences in low-fertility countries. It has become particularly important in the EU members states that host sizeable foreign-born populations, and that are seeing sustained immigration from third countries. In these states, women of reproductive age have become increasingly diverse in terms of their ethnicity, region of origin, religion, and other relevant ethno-cultural characteristics. This diversity could have lasting long-term effects on demographic trends and population composition (Coleman 2006). Across the EU countries, growing shares of births are to mothers of immigrant origin. In some EU member states (MS), the percentage of live births to immigrant mothers has exceeded $25-30 \%$ over the past decade. ${ }^{2}$ While births to foreign-born mothers have increased, the net effect on overall TFR remains limited, though not negligible (Sobotka 2008).

As the heterogeneity of European populations is growing, immigrants and their descendants could shape demographic trends to a greater extent in the future than they do today, assuming the current patterns of childbearing and of immigration persist. While immigrants tend to have higher fertility than the native-born population, fertility rates vary greatly among immigrant women depending on their country of origin (Andersson 2004; Dubuc 2012; Mussino and Strozza 2012; Sobotka 2008). In general, women from low-fertility countries tend to have low fertility in the host country, and often have even lower fertility than native-born women; whereas women from high-fertility countries tend to have high fertility in the host country (Mussino and Strozza 2012; Persson and Hoem 2014; Kulu et al. 2017). However,

2 See EUROSTAT table: demo_facbc 
the country of origin may be a less important explanatory variable than the selection of immigrants in terms of their individual characteristics (Andersson 2004; Kulu 2005). Women's educational attainment, as well as their reasons for migration, likely influence their fertility.

A growing number of studies have highlighted that the country of origin, the age at arrival, and the duration of stay in the host country affect the underlying differences in fertility levels between immigrants. There is strong evidence that the fertility levels of migrants are converging with those of the native-born population. However, for some specific immigrant groups within specific country contexts, the fertility levels of the descendants remain well above the fertility levels of the nativeborn population (Dubuc 2012; Kulu et al. 2019). Convergence towards mainstream fertility levels is observed across different generations of immigrants, with firstgeneration immigrants (G1) having the highest fertility rates, those who arrived as children (G1.5) having lower fertility rates, and the descendants of immigrants (G2) having fertility rates that are the most similar to those of native-born women (Kulu et al. 2017; Krapf and Wolf 2016). This pattern of intergenerational convergence has been interpreted through the lens of the adaptation hypothesis, which assumes that the context of the host country greatly influences an individual's fertility desires, plans, and behaviours. In contrast, the socialisation hypothesis posits that each individual's preferences are relatively stable over his/her lifetime, and depend on the socialisation context. Thus, according to this hypothesis, an immigrant's age at arrival, and whether s/he was socialised in a high- or a lowfertility context, will influence his/her observed fertility. Dubuc (2012) found that the convergence, which is prominent for populations originating from high-fertility countries, reflects in part decreasing fertility in sending countries, and in part intergenerational adaptation to the UK mainstream.

The adaptation hypothesis argues that immigrants' intentions and behaviours change in response to the host country context. Looking at Sub-Saharan African immigrants in France, Afulani and Asunka (2015) found more evidence for the adaptation hypothesis than for the socialisation hypothesis. They also showed that the adaptation hypothesis accounts for the immigrants' actual fertility, rather than just their fertility ideals.

Fertility is closely intertwined with the migration process. The disruption hypothesis posits that childbearing is likely to be depressed around the time of migration, or may not have started prior to immigration. Consequently, fertility often peaks in the first years after arrival in the host country, resulting in a tempo effect that influences period measurements of immigrant fertility (Toulemon 2004; Mussino and Strozza 2012; Robarts et al. 2015). Persson and Hoem (2014) found for Sweden that the disruption hypothesis is particularly relevant for non-EU immigrants from low-income countries, while non-EU immigrants from high-income countries tend to delay childbearing until several years after migration. Robarts et al. (2015) confirmed both findings for UK immigrants, and showed that (a) the fertility rates of immigrant women from low-income countries peak within four years 
after immigration; and that (b) women from high-income countries tend to delay childbearing and have lower fertility rates.

The hypothesis of interrelated life events explains the high fertility upon arrival in the host country in the context of the family formation process. According to this hypothesis, immigrants who arrive on family reunification grounds exhibit high fertility in the years immediately after arrival, because for them, childbearing is directly linked to immigration (Castro Martin and Rosero-Bixby 2011; Mussino and Strozza 2012; Robarts et al. 2015). In contrast, women who arrive in the country to work or to study are expected to delay childbearing and have lower fertility. A decision to have a child shortly after arriving in the host country can also be linked to poor employment prospects (Kulu and Milewski 2007). The interrelation between the opportunities in different life domains influences a couple's fertility decisions.

As immigrants arriving on various grounds can also differ in their educational characteristics, education becomes an important confounding variable to account for when examining fertility differentials. Dubuc (2017) found that educational differences between successive generations of immigrant women and their daughters indeed explained in part the convergence in fertility towards the mainstream.

Thus, making assumptions about the future fertility of immigrant women is challenging, because we would have to account for (a) ongoing fertility transitions in developing countries and (b) educational expansion, and (c) make assumptions about the composition of future migration flows by country of origin. Explicit and implicit migration policies can affect the future composition of immigrants to the EU in terms of their grounds for immigration, their countries of origin (e.g., restrictions on family reunification that favour already established diaspora), their educational levels (e.g., selective policies favouring highly educated immigrants with skills needed in the future labour market), or their gender (e.g., the demand for care workers in ageing societies). Due to selection, immigrants may exhibit very different childbearing patterns than those of the mainstream in the country of origin, as they may be arriving from high-/low-fertility regions, and they could be selected in terms of their educational characteristics.

To conclude, existing evidence of fertility differentials between immigrants and the native-born population, as well as across various immigrant groups, highlights the importance of accounting for three main variables associated with such differentials: the country of origin, the age at arrival in the host country, and the duration of stay. To illustrate the effect of modelling with different sets of differentials - while easing the interpretation of the results - we keep the composition of immigrants, ${ }^{3}$ as well as fertility differentials among the migrant groups, constant for the whole

\footnotetext{
3 The composition of non-EU immigrants is estimated using the EU Labour Force Survey, adjusted using data from the 2011 census on country of origin and year of arrival. This process results in $39 \%$ of these immigrants coming from Asia; 35\% coming from Africa or MENA; $11 \%$ coming from Latin America; and 15\% coming from a non-EU European country, North America, or Australia.
} 
projection period. As it is well-documented that enrolment in education has a postponement effect on fertility, we argue in the next section that enrolment in education should be accounted for alongside educational gradients in fertility.

\section{Data and methods}

\subsection{Approach and scenarios}

Our aim is to explore the impact of modelling fertility differentials by education from a life course perspective rather than relying on the conventional approach used in the multistate population projections, and to examine the effects of differentials between the native-born population and various groups of immigrants on projection results. To identify such differences, we build our scenarios around assumptions regarding future overall fertility (TFR) ${ }^{4}$ and educational gradients in fertility rates drawn from existing multistate population projections by education (Lutz et al. 2014 and 2018). Before we describe the scenarios and their underlying assumptions, we outline the methodological challenges we face in modelling fertility differentials.

In a conventional multistate approach, educational differentials in fertility are modelled as if women at a given age had completed the highest level of education they would ever achieve. This approach is problematic in high-income countries, in which a large share of a given female cohort is studying at the post-secondary level. For example, at ages 20-24, most women will have completed upper secondary education, but a large proportion of them will be enrolled in post-secondary education. In Lutz et al. (2014 and 2018), educational differentials in fertility are modelled with fertility rates that are derived from the cohort fertility rates of women who have not studied beyond the upper secondary level. Education is strongly associated with fertility timing, and in most economically advanced societies, the birth of the first child is closely linked to other life transitions, with the completion of full-time education being a strong normative determinant (Mortimer et al. 2005). Consequently, the fertility rates of women in full-time education are much lower than they are among women who have completed their education.

This methodological issue becomes highly relevant when the modelling of fertility described above is coupled with the assumption that educational expansion is continuing. In the global educational trend (GET) scenario, it is assumed that an ever-larger share of women will pursue post-secondary education in each subsequent projected cohort (Barakat and Durham 2014). Thus, the share of students among women of younger reproductive ages increases. In such cases, the conventional modelling of education differentials leads to overestimated fertility rates because the fertility rates of women with levels of completed education are

\footnotetext{
4 The assumptions are based on extensive information from experts, and are detailed in Basten et al. (2014). The fertility assumptions in our EDU scenario are drawn from the SSP2 scenario.
} 
applied to women who are still in full-time education. This results in a higher number of projected births to women with lower and upper secondary education. We expect this bias to become increasingly pronounced towards the projection horizon because of the projected educational expansion.

A solution lies in modelling fertility differentials from a life course perspective. Microsimulation models are well-suited to addressing this shortcoming of multistate models, as they simulate the life courses of individuals across multiple life careers. In the CEPAM-mic microsimulation model (detailed in the next section), we go beyond modelling fertility by educational attainment only by introducing a new status - STUDENT - and include fertility differentials between women enrolled in full-time education and women who have completed their education.

The fertility module is set up in a flexible way that allows us to gradually introduce changes to how we model fertility differentials. We have built six scenarios combining three assumptions of fertility and two assumptions of migration (Table 1). Our baseline scenario (EDU) models fertility in the same way as conventional multistate models. We include fertility assumptions (age- and education-specific fertility rates) from the medium scenario of the human capital projections of Lutz et al. (2018). Then, we introduce the differences between women in fulltime education (students) and women with completed education. We label this scenario EDU + STUDENT. Next, we add fertility differentials between native-born and immigrant women with different characteristics, and call this scenario EDU + STUDENT + IMMIG. All dimensions of the migration status variable (IMMIG) are detailed in section 3.3.

In order to test the sensitivity of projection results to different possible future immigration flows, we run the same three scenarios with modified levels of immigration to the EU (Table 1). The high migration assumption is derived from the Canadian example - a country with a high immigration rate and a selective migration policy. If the immigration of non-EU nationals increased to the Canadian rate, we would see a doubling of the projected inflow of immigrants from outside of the EU relative to recent trends. The doubling of immigration levels is coupled with greater emigration outflows given the constant emigration rates. Migration between the EU member states is modelled separately using a matrix of bilateral migration rates that are held constant until 2060. Future mortality assumptions are taken from Lutz et al. (2014), and are identical in all scenarios.

Comparisons between the EDU and EDU + STUDENT scenarios allow us to evaluate differences in the projection results that arise due to methodological differences in the modelling of education differentials. The differences between the latter scenarios and the scenario with migrant characteristics illustrate the effect of including population heterogeneity due to migration that is not accounted for in the human capital projections (Lutz et al. 2014, 2018). Our migration assumptions lead to increasing shares of non-EU immigrants in the EU population. We expect to find that the effect of migrant fertility differentials becomes increasingly important towards the projection horizon, and becomes more pronounced in the high immigration scenarios in which we double immigration to illustrate the effect 
Table 1:

Overview of scenarios and assumptions

\begin{tabular}{|c|c|c|c|c|c|}
\hline \multirow[b]{2}{*}{ Scenario } & \multicolumn{5}{|c|}{ Scenario assumptions: } \\
\hline & Fertility & $\begin{array}{l}\text { Immigration } \\
\text { from outside } \\
\text { the } E U\end{array}$ & $\begin{array}{l}\text { Emigration } \\
\text { outside } \\
\text { the EU }\end{array}$ & $\begin{array}{l}\text { Intra-EU } \\
\text { migration }\end{array}$ & Mortality \\
\hline EDU & $\begin{array}{l}\text { Medium (by } \\
\text { educational } \\
\text { attainment) }\end{array}$ & cca 10 million & Average rate & Average in & Medium, by \\
\hline EDU + STUDENT & $\begin{array}{l}\text { Medium + differentials } \\
\text { for student status }\end{array}$ & every 5 years & 2013-2016 & $\begin{array}{l}\text { and out } \\
\text { rates }\end{array}$ & education $^{* *}$ \\
\hline $\begin{array}{l}\text { EDU + STUDENT } \\
\text { + IMMIG }\end{array}$ & $\begin{array}{l}\text { Medium + student } \\
\text { status + migrant } \\
\text { status }\end{array}$ & & & 2009-2016 & \\
\hline $\begin{array}{l}\text { EDU High } \\
\text { migration }\end{array}$ & $\begin{array}{l}\text { Medium (by } \\
\text { educational } \\
\text { attainment) }^{*}\end{array}$ & cca 20 million & Average rate & Average in & Medium, by \\
\hline $\begin{array}{c}\text { EDU + STUDENT } \\
\text { High migration }\end{array}$ & $\begin{array}{l}\text { Medium + differentials } \\
\text { for student status }\end{array}$ & every 5 years & 2013-2016 & $\begin{array}{l}\text { and out } \\
\text { rates }\end{array}$ & education $^{* *}$ \\
\hline $\begin{array}{l}\text { EDU + STUDENT } \\
+ \text { IMMIG High } \\
\text { migration }\end{array}$ & $\begin{array}{l}\text { Medium + student } \\
\text { status + migrant } \\
\text { status }\end{array}$ & & & 2009-2016 & \\
\hline
\end{tabular}

Notes: * Lutz et al. (2018)

** Caselli et al. (2014)

of migration. The presented scenarios illustrate what would happen if fertility differentials remained at recent past levels. We also assume that the pace of convergence towards native-born fertility levels with the duration of stay will remain the same as in was the recent past. ${ }^{5}$ If future immigrants have lower fertility after arrival, if the convergence occurs more quickly, or if the composition of immigrants changes significantly, the effects of these scenarios on overall fertility and the number of births would be less pronounced than our results indicate.

\subsection{CEPAM-Mic microsimulation model}

CEPAM-mic is a microsimulation projection model covering $28 \mathrm{EU}$ countries that has been developed within the framework of CEPAM project. The model was developed in the Modgen language, and built following the framework for the microsimulation models developed by the Laboratoire de Simulation Démographique (LSD) of the Canadian National Institute of Scientific Research (Bélanger et al. 2019).

\footnotetext{
5 For the descendants of immigrant parents (second generation), we assume the same fertility rates at
} a given educational level as we do for the EU-born population. 
In addition to taking into account age, sex, and educational attainment, ${ }^{6}$ the baseline population is structured by region of birth, ${ }^{7}$ duration or residence $(0-4$, $5-9,10+$ years), age at migration $(0-14,15+)$, student status (a student in fulltime education, not a student), and several other characteristics not utilised in our analysis, such as language and labour force participation status (Sabourin et al. 2017). The baseline population is derived from the Labour Force Survey, the European Social Survey, and the 2011 census, and it is calibrated to the 2015 base population of CEPAM-mac by age, $\operatorname{sex}^{8}$ education, and EU country (Lutz et al. 2018). The method is described in detail in Sabourin et al. (2017).

CEPAM-mic uses exactly the same mortality rates by country, age, sex, and education as those used in the medium scenario of Lutz et al. (2014). These rates were determined after consultation with an expert panel, and are detailed in Caselli et al. (2014). The number of international immigrants from non-EU countries is estimated at about 10 million immigrants every five years, which corresponds to an average of two million immigrants annually during 2013-2016. This inflow is assumed to remain constant in every five-year step of the simulation, but is doubled to 20 million in the scenarios using a high migration variant.

The out-migration rates to non-EU countries are estimated using the average number of out-migrants from 2013 to $2016^{9}$ divided by the average population aged $20-34^{10}$ during the same period, by country. First, the Eurostat-derived outmigration rates are applied to the exposure of the population aged 20-34 in order to obtain the expected number of overall out-migrants in a given year. Age-specific outmigration rates are computed by taking the ratio of out-migrants to the population by age, sex, and country of residence. The age patterns of both immigrants and emigrants follow the standard Rogers-Castro schedule (Rogers and Castro 1981), with the highest levels of migration being observed among the young adults in their twenties.

Out-migration rates in the simulation are recalculated every five years. During the simulation, out-migrants can move within the EU and be assigned a new country of residence, or they can leave the EU and be removed from the simulation. The

\footnotetext{
6 Educational attainment: Low $=$ lower secondary or below (ISCED $0-2$ ), Middle $=$ upper secondary (ISCED 3), High = post-secondary (ISCED 4-8); ISCED 2011 definitions.

7 Regions: own country, EU-15 (old member states), NMS-13 (new member states, since 2004), Europe outside EU-28, North Africa, Other Africa, Near Middle East, East Asia, South and South-East Asia, North America, Oceania, Latin America.

8 The population composition of the EU countries by age and sex in 2015 corresponds to the that of EUROSTAT (table: demo_pjan).

9 EUROSTAT table: migr_emi2

10 The age bracket 20-34 is chosen based on empirical data showing that most out-migrants fall within this age bracket. Using total population in the exposure affects out-migration rates, especially in Southern European countries, due to the older age composition and low migration rates among the older age groups. Using only ages at which the propensity to migrate is highest yields more comparable results.
} 
proportion of out-migrants leaving the EU is estimated from Eurostat tables on emigration according to the region of destination. ${ }^{11}$

The origin-destination matrix for intra-European migration was derived using an update for the period 2009-2016 of Raymer et al.'s (2013) Bayesian estimates of European migration. Country-specific calibration factors are then calculated from a preliminary simulation for the period 2011-2015 in order to obtain the same number of entrances by country as those estimated by Eurostat for the same period. These calibration factors are kept constant for the rest of the projection.

The education module, which is exhaustively described in Marois et al. (2019), determines the highest completed level of education and the age at graduation (for the highest level as well as for intermediary levels) of an individual at birth, considering cohort trends as well as differentials for the most relevant determinants of the individual's education: mother's educational attainment, religion, language, and place of birth (for immigrants who arrived as children). Existing research shows strong intergenerational transmission of education, with mother's education being a particularly salient determinant (Shavit and Blossfeld 1993; Hertz et al. 2008). Empirical data used to estimate education parameters revealed that immigrants who speak a non-European language and who are from some specific regions face disadvantages in access to post-secondary education, even after controlling for mother's education (Marois et al. 2019). Individuals are set as students starting from age five until the age of graduation from the highest completed level of education (up to age 29). Thus, the modelling of education allows us to distinguish, for each individual at each projection step, the highest level of education that the individual will reach during his/her life course, his/her current level of education, and whether or not $\mathrm{s} / \mathrm{he}$ is still in schooling.

\subsection{CEPAM-mic fertility module}

The fertility module utilises the age- and education-specific fertility rates of the medium scenario of the human capital projections (Lutz et al. 2018, termed the baseline scenario in this paper), and is built in a flexible way that allows us to implement educational attainment gradients also differentials by student status and migrant background characteristics. These additional parameters are estimated using a logit model that is detailed below. All analyses of Labour Force Survey data (LFS) are executed in STATA 15.1.

We use annual data from the Labour Force Survey 2014-2016 12 that include information on 5,300,110 women of reproductive age (15-49) and 203,113 coresident children younger than one year old. LFS is the largest household survey

\footnotetext{
11 EUROSTAT table: migr_emi3nxt

12 Annual data from publicly available microdata files for scientific use requested from the EUROSTAT.
} 
that includes a set of harmonised variables for all $28 \mathrm{EU}$ member states. This scope is both an advantage and a disadvantage, because it comes at the price of a reduced level of detail, since some countries provide the data in more aggregated categorisations than others. This lack of detail has also affected some of our variables of interest. For example, the region of birth of the respondent is categorised according to 21 broad, predefined regions, some of which conceal important differences in fertility.

To create our dependent variable, we match the co-resident youngest child aged zero with the mother in the household using the mother identifier. ${ }^{13}$ In the LFS dataset, only the age of the youngest child is available in single units of age, while the ages of all other household members are coded in five-year age groups. We use a logit model to estimate the odds ratios of giving birth to a child for women with different educational ${ }^{14}$ and migrant characteristics. Our response variable is whether a woman has a child younger than one year old (yes/no).

The logit model, which is expressed in equation (1), aims at estimating differentials for student status $\left(\beta_{9}\right)$ and migrant status $\left(\beta_{8}\right)$ net of age and education. Thus, we include in the model interaction variables that control for age-, education-, and country-specific ( $\beta_{0}$ to $\left.\beta_{7}\right)$.

$$
\begin{aligned}
\operatorname{logit}(P)= & \beta_{0}+\beta_{1} A G E+\beta_{2} E D U+\beta_{3} C N T R Y+\beta_{4}(A G E * E D U) \\
& +\beta_{5}(A G E * C N T R Y)+\beta_{6}(E D U * C N T R Y) \\
& +\beta_{7}(A G E * E D U * C N T R Y)+\beta_{8} I M M I G+\beta_{9} S T U D E N T
\end{aligned}
$$

where AGE is age in five-year age groups (15-49); EDU is educational attainment (low, medium, and high); CNTRY is a country (28 EU member states); STUDENT indicates enrolment in full-time education (yes/no, only for ages 15-29); and IMMIG is a composite migration variable constructed using region of birth, age at arrival in the host country, and duration of stay in the host country. We assign the regions of birth of immigrants to six broad regional categories: EU+ (EU28, ${ }^{15}$ EFTA $^{16}$ countries, Northern America and Australia), Eastern Europe and Turkey,

\footnotetext{
13 Variable HHMOTH. Most young children tend to live in the same household as their mother. One potential bias of this matching procedure is that in more complex, multigenerational households, the child may not be matched or wrongly matched to a woman who is not his/her biological mother.

14 The model uses highest educational attainment at the time of the survey. The results may be subject to reverse causality in terms of education.

15 EU28 countries as of 2019, including the United Kingdom: AT, BE, BG, CZ, CY, DE, DK, EE, EL, ES, FI, FR, HR, HU, IE, IT, LV, LT, LU, MT, NL, PL, PT, RO, SI, SK, SE, UK.

16 EFTA (European free trade area) includes the following non-EU countries: Iceland, Liechtenstein, Norway, and Switzerland.
} 
Africa and MENA, ${ }^{17}$ Asia (excluding the Middle East), and Latin America. ${ }^{18}$ The level of aggregation of the variable in the LFS does not allow us to separate Turkey from Eastern Europe or Northern Africa from sub-Saharan Africa, even though such distinctions would be very useful for capturing fertility differentials. Age at arrival in the host country differentiates between those who arrived as children (by age 15) and are called generation 1.5 (G1.5), and those who arrived as adults and are called first-generation immigrants (G1). This distinction is important, as it helps us capture the extent to which socialisation in the host country affects family formation and fertility in immigrant populations. First-generation immigrants are further split by the duration of stay in the host country ( $0-4$ years, five or more years) in order to capture potentially elevated fertility rates after the time of arrival (tempo effect).

The resulting migration variable is coded into 16 categories:

- native-born,

- G1 EU+ immigrant, in the country for less than five years,

- G1 EU+ immigrant, in the country for more than five years,

- G1.5 EU+,

- and so on for the other four regions of birth.

Figure 1 illustrates the odds ratios derived from $\beta_{8}$. They show that even after controlling for age and education, immigrant women from outside the EU+ tend to have higher fertility than native-born women. As expected, the results indicate that first-generation (G1) immigrants who only recently arrived in the host country have the highest risk of having a young child, probably due to a period effect after migration that arises from the interrelation of life events, and that is also influenced by the reasons for migration. The odds are lower for G1 women resident in the host country for at least five years, but these women still have higher odds than those who arrived as children (by age 15, G1.5). This finding is again consistent with the existing studies, and is usually interpreted in the context of the socialisation and adaptation hypotheses. The differentials are particularly pronounced for women from Africa and MENA, who have significantly higher odds. This is the case even for G1 immigrants with a longer duration of stay and for G1.5 immigrants, although the gradient is as expected, and the odds decrease. For Asian women, we can see that G1.5 immigrants do not differ significantly from their native-born counterparts.

17 MENA stands for Middle East and North Africa.

18 Not all of the 28 member states provide the data coded into all 21 detailed regions. Immigrants from EFTA, North America, and Australia are grouped with EU28 migrants, as they have similar fertility levels. We did not add them to Eastern Europe + Turkey because the differentials in this region are clearly determined by Turkish women. Germany does not provide data on the country of birth for those not born in Germany. Therefore, we have imputed the foreign country of birth using citizenship information (imputed 55\% of missing cases). Naturalised immigrants with German citizenship are excluded from the analysis. Of the imputed cases, $45 \%$ were resident in Germany less than 10 years, compared to $6.5 \%$ for naturalised immigrants. Given that fertility peaks in the years immediately after migration, the exclusion of naturalised immigrants should not greatly alter the results. 
Figure 1:

Odds of having a child by region of origin, generational status, and duration of stay, native-born $=$ reference category

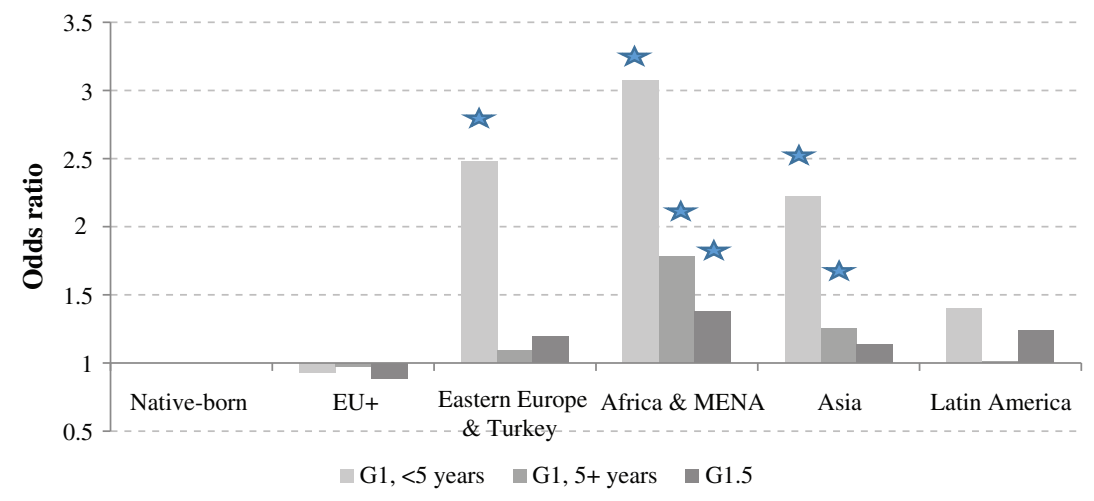

Note: Statistically significant results at the 0.05 level marked by an asterisk.

Source: LFS 2014-2016 yearly data, publicly available scientific datasets.

For women from Eastern Europe and Turkey, we observe significantly higher odds of having a young child for recent G1 immigrants only. Immigrant women from other EU+ countries do not differ significantly from native-born women.

In the microsimulation, we are simulating the life courses of individuals in continuous time. ${ }^{19}$ The advantage of this approach is that it enables us to link a woman's fertility to stages in her educational career, as a woman who is still in education is less likely to have a child than a woman with completed education, even though the actual educational attainment levels of these women are the same at a given age. The parameter for the student status reduces fertility for women who are in full-time education. Looking at the results of the regression model, we can see that a woman who is in education has about 10 times lower odds of having a child than a woman who has completed her education (odds ratio of 0.123 after controlling for control variables).

In order to use in the microsimulation the baseline rates by age and education of Lutz et al. (2018), the parameters for the student status $\left(\beta_{9}\right)$ and the migration status $\left(\beta_{8}\right)$ are compared to the weighted population average (using the option contrast gw in STATA 15.1). ${ }^{20}$ For instance, the odds ratio for a student compared to the weighted average population changes from 0.123 to 0.168 ; and, conversely, an odds ratio of 1.365 is applied to the non-student (again, compared to the weighted average

19 The modelling of fertility from a period perspective does not necessarily reflect the cohort's life course behaviour, particularly for immigrant women who may have had children prior to arrival.

20 LFS dataset is reweighted by the base population of the microsimulation model in order to use identical reference populations. 
population). In the fertility module, parameters $\beta_{0}$ to $\beta_{7}$ (control variables, age and education) are replaced by the age- and education- specific fertility rates by country taken from the baseline scenario. The EDU scenario simply replicates the fertility rates by age and education of CEPAM-mac by switching $\beta_{8}$ and $\beta_{9}$ to zero. In the second EDU + STUDENT scenario, we switch on the $\beta_{9}$ parameter, and thus add the differentials by student status to the model. In the third EDU + STUDENT + IMMIG scenario, we switch on the migration status $\left(\beta_{8}\right)$ and obtain the full model. These steps are repeated for the high migration scenarios. In all of these scenarios, the parameters for student status and migrant status are held constant.

\section{Results}

The resulting TFRs for the EU28 in the projection scenarios are shown in Figure 2. The increases in the TFRs found for all scenarios result from the general trends set in the assumptions of the reference scenario of Lutz et al. 2018, for more details, see also Basten et al. 2014). The baseline EDU scenario (yellow line) assumes that the TFR increases from 1.65 in 2015-19 to 1.85 in 2050. The differences between the EDU scenario and the additional scenarios reflect the impact of accounting for heterogeneity in fertility behaviours with respect to education and migration, and the variations in the composition of women of reproductive age. If we consider all differentials (by educational attainment, for students and for immigrants), we arrive at lower TFRs that peak at 1.79 children per woman. This result is driven by educational expansion, as illustrated by the EDU + STUDENT scenario that projects a TFR of 1.71 in 2040-59. The projected TFRs in the EDU + STUDENT scenario are lower because in this scenario, the shares of young women enrolled in higher education who have very low fertility while enrolled are increasing. Once we differentiate between the native-born women and various groups of immigrant women (EDU + STUDENT + IMMIG scenario), the overall TFR increases, but to a lower level than in the EDU scenario.

The gap in the fertility levels of EU-born women and foreign-born women is caused by both the educational composition and the migrant characteristics of foreign-born women (Figure 3). In the EDU + STUDENT scenario, in which we do not account for migrant characteristics, foreign-born women have 0.14 to 0.19 points higher TFRs than the TFRs of higher educated EU-born women. In the EDUSTUDENT scenario, the TFRs depend only on the educational composition and the share of women in education.

Once we account for fertility differences across various groups of foreign-born women, the estimated TFR of the foreign-born women increases to 2.5-2.6. The estimated TFR of the EU-born women is about $1.65^{21}$ (EDU + STUDENT + IMMIG scenario in Figure 3).

21 Includes both native-born and EU-mobile female citizens. 
Figure 2:

Past and projected total fertility rates (TFR) for the EU28 in four selected scenarios

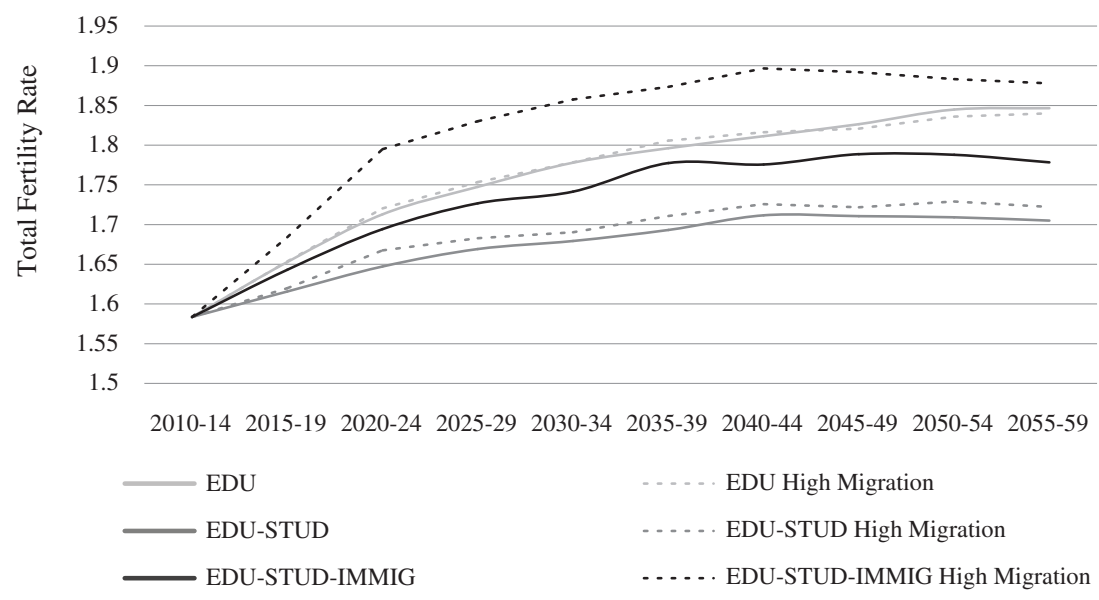

Source: EUROSTAT (2010-2014) and CEPAM (2015-2060)

Figure 3:

Estimated TFRs of EU-born and foreign-born women by scenario

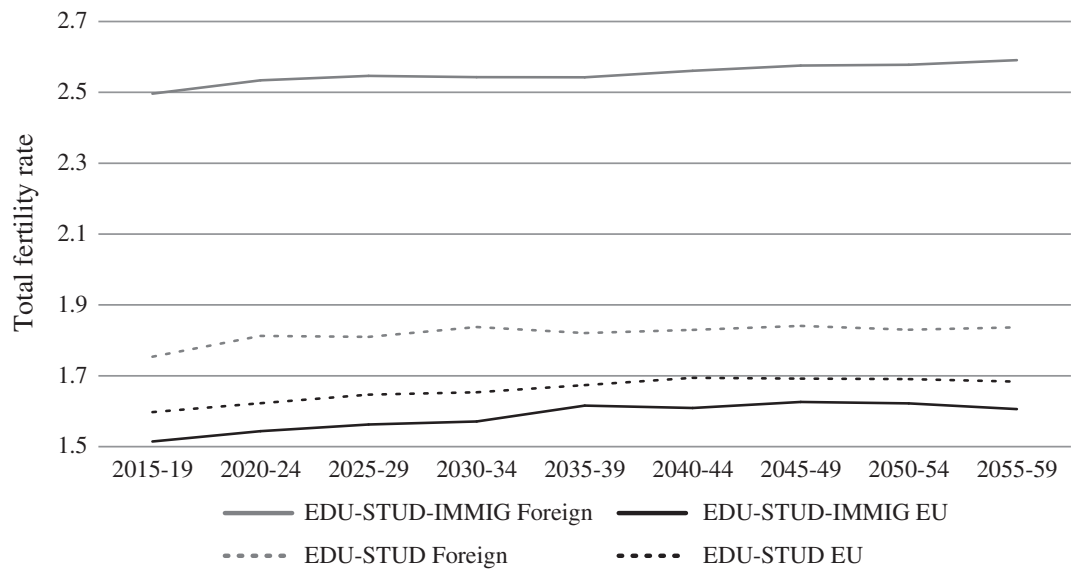

Source: CEPAM (2015-2060)

The impact of the fertility patterns of foreign-born women on overall fertility depends on the volume of migration and the stock of foreign-born women of fertile age in the baseline population. If both the fertility differences between native-born and foreign-born women and the current migration flows persist at recent levels, 
the overall TFRs in the EDU scenario and in the EDU + STUDENT + IMMIG scenarios turn out to be very similar. The net effect of immigrant fertility on the overall TFR increases from 0.12 points in 2015-19 to 0.17 points in 2055-59 (Table 2). At current fertility levels, a two-fold increase in immigration from nonEU countries results in a rapidly increasing overall TFR peaking at 1.9 in 2040-44. In the high migration scenario, the net effect of immigrant fertility rises to 0.25 in 2055-59 (Table 2). This finding reflects the rapidly increasing population of immigrant women in the EU population, which is projected to rise from an estimated 10.4 million in 2015 to 38.1 million in 2055 under high migration assumptions, but to just 19.3 million under baseline migration assumptions (Table 3). The share of immigrant women of reproductive age is projected to increase from $10.5 \%$ in 2015 to about $30 \%$ in 2060 in the high migration scenario, but to only $20 \%$ in 2060 in the scenarios with baseline migration (Table 3 ).

The compositional effects of increased immigration on the overall projected TFRs are more pronounced in countries with larger stocks and projected flows of immigrants from third countries, some of which are listed in Table 2. At current differentials, the net effects of immigrant fertility are projected to be the most pronounced in Sweden, with the projected share of foreign-born women of reproductive age expected to increase from $23 \%$ in 2015 to $30 \%$ in the baseline migration scenario, and to $39 \%$ in the high migration scenario.

In the scenarios with baseline migration, the projected number of births declines from an estimated 26.0-26.5 million in 2015-19 to 23.1-25.7 in 2055-59 (Figure 4). These decreases occur despite the assumed increases in the TFRs (Figure 2) because the number of women of reproductive age declines from 112 million in 2015 to 97 million in 2055 (Table 3), even at sustained recent past immigration volumes. In the doubled migration scenario, the projected population of women of reproductive age increases to 126 million in 2055. In combination with current fertility differentials, this results in a steadily increasing projected number of births in all of the high migration scenarios (Figure 4). When we compare the EDU and the EDU high migration scenarios, we see that a doubling of migration results in $2 \%$ more births in 2015-19, with a gradual upward trend to $28 \%$ more births in 2055-59. In the EDU + STUD + IMMIG scenarios, larger increases in immigration result in 5\% more births as early as in 2015, and 41\% more births in 2055 (34.9 million vs. 24.8 million, see also Table 4).

In the EDU scenarios, the number of births is boosted by additional births to students who are assumed to have the same fertility rates as their peers with completed education. The difference in the projected number of births in the EDU and EDU + STUDENT scenarios gradually increases from $2 \%$ to $10 \%$ over the projection period. The EDU + STUDENT scenario assumes that there are no fertility differentials between native-born and foreign-born women with the same educational levels. If this is indeed the case, and the immigration flows continue to be of magnitudes similar to those in the recent past, the projected number of births will fall below 24 million by 2060 . 


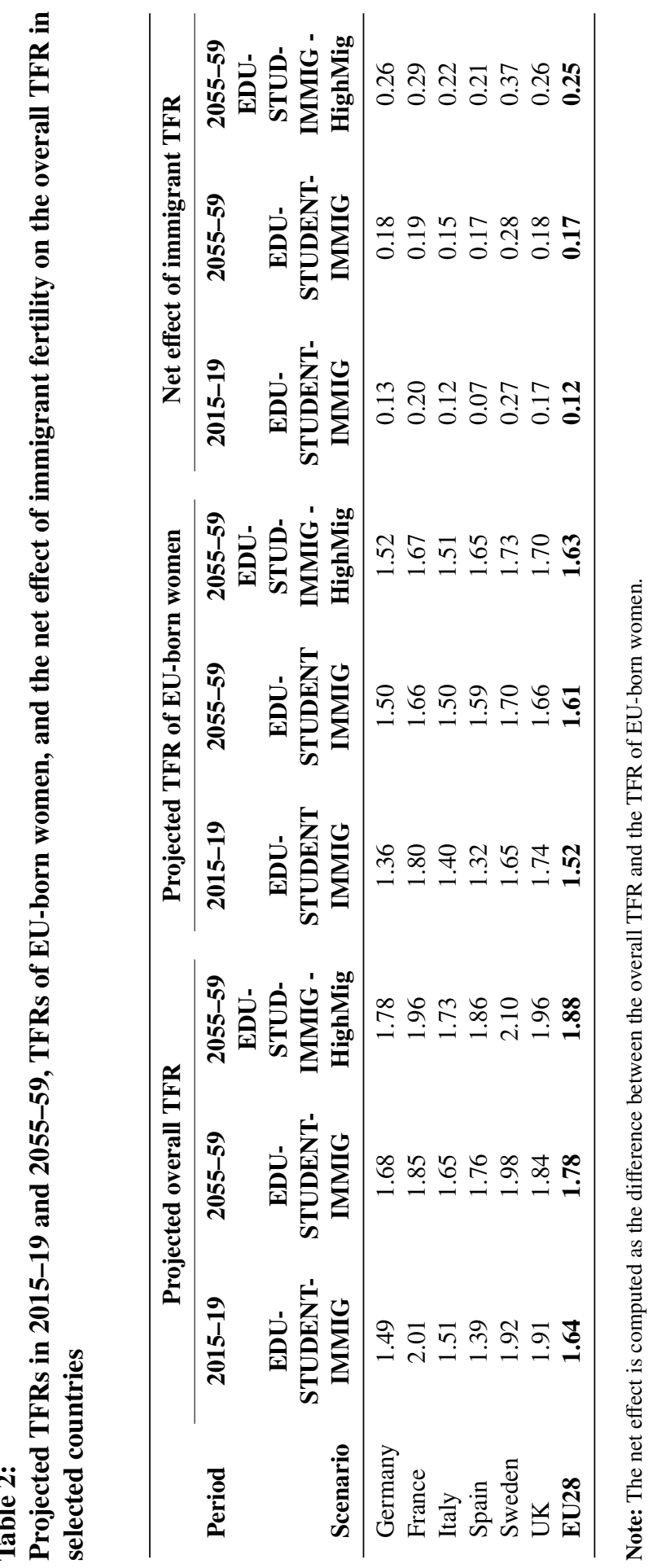


Table 3:

Projected size and composition of women of reproductive age in EU28

\begin{tabular}{lccccc}
\hline & $\mathbf{2 0 1 5}$ & $\mathbf{2 0 2 5}$ & $\mathbf{2 0 3 5}$ & $\mathbf{2 0 4 5}$ & $\mathbf{2 0 5 5}$ \\
\hline & \multicolumn{5}{c}{ Proportion of women aged 15-49 } \\
& \multicolumn{5}{c}{ born outside EU } \\
EDU + STUDENT + IMMIG & $11 \%$ & $16 \%$ & $19 \%$ & $20 \%$ & $20 \%$ \\
EDU + STUDENT + IMMIG High migration & $11 \%$ & $21 \%$ & $28 \%$ & $31 \%$ & $30 \%$ \\
& \multicolumn{5}{c}{ Women of reproductive age } \\
EDU + STUDENT + IMMIG & 112 & 104 & 100 & 97 & 97 \\
EDU + STUDENT + IMMIG High migration & 112 & 112 & 116 & 120 & 126 \\
\hline
\end{tabular}

Source: EUROSTAT (2015) and authors (2020-2055)

\section{Figure 4:}

Projected births in the EU28 in the projection scenarios considering various fertility differentials and immigration assumptions

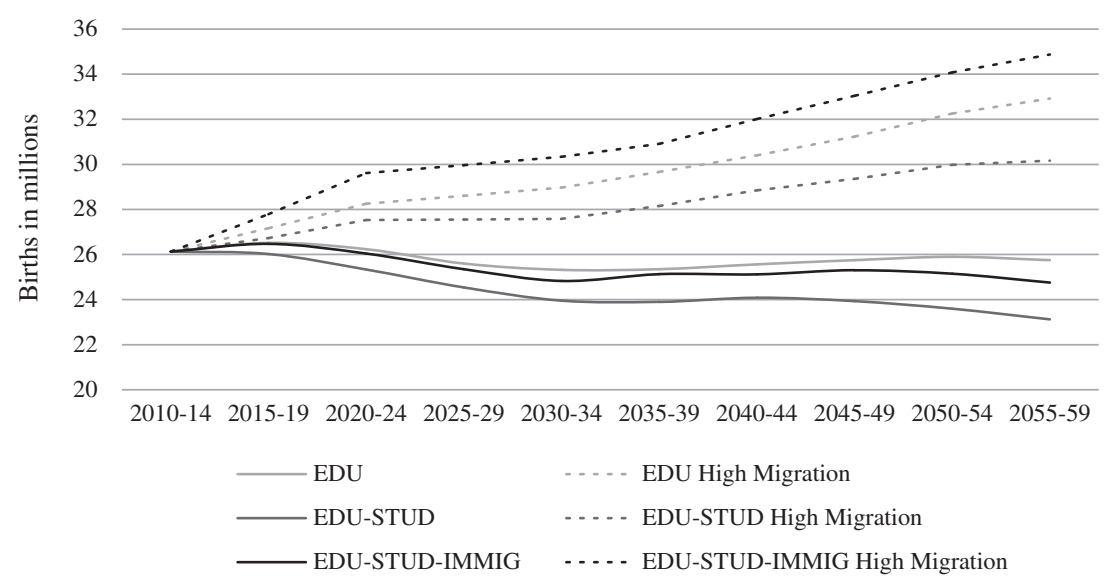

Source: EUROSTAT (2010-2014) and CEPAM (2015-2060)

When we look at the overall TFRS and the projected births in the EDU and EDU + STUDENT + IMMI scenarios, it may appear that modelling fertility with a broader set of differentials does not affect the projection results enough to compensate for the additional complexity in the estimations that need to be performed to arrive at a set of fertility parameters that capture an increased number of dimensions. However, this is the case only if we assume that there is no change in immigration among third-country nationals. A set of scenarios with doubled immigration (dotted lines in Figure 4) show sizeable differences in the projected number of 
Table 4:

Projected births (in millions) in the full model with baseline and double migration, selected countries

\begin{tabular}{|c|c|c|c|c|c|c|}
\hline \multirow{2}{*}{$\begin{array}{l}\text { Scenario } \\
\text { Period }\end{array}$} & \multicolumn{2}{|c|}{ EDU + STUDENT + IMMIG } & \multicolumn{2}{|c|}{$\begin{array}{c}\text { EDU }+ \text { STUDENT }+ \text { IMMIG } \\
\text { high migration }\end{array}$} & \multicolumn{2}{|c|}{ Scenario difference } \\
\hline & 2015-19 & 2055-59 & 2015-19 & $2055-59$ & 2015-19 & 2055-59 \\
\hline Germany & 3.7 & 4.1 & 4.0 & 6.0 & 0.24 & 1.89 \\
\hline France & 4.1 & 3.6 & 4.3 & 5.0 & 0.20 & 1.36 \\
\hline Italy & 2.7 & 2.4 & 2.8 & 3.3 & 0.12 & 0.90 \\
\hline Spain & 2.1 & 1.8 & 2.2 & 2.7 & 0.09 & 0.88 \\
\hline Sweden & 0.6 & 0.8 & 0.7 & 1.4 & 0.07 & 0.51 \\
\hline UK & 4.2 & 4.2 & 4.4 & 6.1 & 0.22 & 1.86 \\
\hline \multirow[t]{3}{*}{ EU28 } & 26.5 & 24.8 & 27.8 & 34.9 & 1.32 & 10.12 \\
\hline & \multicolumn{2}{|c|}{ EDU + STUDENT } & \multicolumn{2}{|c|}{$\begin{array}{c}\text { EDU + STUDENT } \\
\text { high migration }\end{array}$} & \multicolumn{2}{|c|}{ Scenario difference } \\
\hline & 2015-19 & $2055-59$ & 2015-19 & $2055-59$ & 2015-19 & 2055-59 \\
\hline Germany & 3.6 & 3.7 & 3.8 & 5.1 & 0.13 & 1.37 \\
\hline France & 4.1 & 3.5 & 4.1 & 4.4 & 0.10 & 0.94 \\
\hline Italy & 2.6 & 2.2 & 2.7 & 2.8 & 0.06 & 0.64 \\
\hline Spain & 2.1 & 1.7 & 2.1 & 2.4 & 0.06 & 0.68 \\
\hline Sweden & 0.6 & 0.8 & 0.6 & 1.1 & 0.04 & 0.35 \\
\hline UK & 4.1 & 4.0 & 4.2 & 5.2 & 0.12 & 1.22 \\
\hline EU28 & 26.0 & 23.1 & 26.7 & 30.2 & 0.7 & 7.0 \\
\hline
\end{tabular}

Source: Authors' computations.

births depending on how the fertility differentials are modelled. Similarly, countryspecific results for selected EU countries that are major destination countries of immigrants presented in Table 4 show, with the exception of Germany, a decline in the number of projected births in the EDU + STUDENT + IMMIG scenario with baseline migration, but a sizeable increase in the scenario with doubled migration and at constant fertility differentials. In the case of Sweden, the high migration EDU + STUDENT + IMMIG scenario results in a doubling of the number of births in 2055-59 compared to in 2015-2019. If differences in fertility between the EUborn and the native-born mothers are not taken into account, and only educational differentials are included (EDU + STUDENT), the overall number of births is lower and the surplus in the EDU + STUDENT high migration scenario is smaller than in the full model.

This sensitivity analysis shows that the simple modelling of fertility in the EDU scenario coincidentally captures the effect of the higher fertility rates of immigrant women through the higher fertility rates of women in education at younger reproductive ages. The majority of immigrants arrive as young adults and 
Table 5:

Projected births to non-EU-born mothers (in \%)

\begin{tabular}{lccccc}
\hline & \multicolumn{5}{c}{ Proportion of births to foreign-born mothers (non-EU) } \\
\cline { 2 - 6 } Scenario & $\mathbf{2 0 1 5 - 1 9}$ & $\mathbf{2 0 2 5}-\mathbf{2 9}$ & $\mathbf{2 0 3 5 - 3 9}$ & $\mathbf{2 0 4 5 - 4 9}$ & $\mathbf{2 0 5 5 - 5 9}$ \\
\hline EDU & $14 \%$ & $18 \%$ & $19 \%$ & $19 \%$ & $19 \%$ \\
EDU + STUDENT & $15 \%$ & $19 \%$ & $21 \%$ & $21 \%$ & $21 \%$ \\
EDU + STUDENT + IMMIG & $20 \%$ & $26 \%$ & $27 \%$ & $27 \%$ & $28 \%$ \\
EDU + STUDENT + IMMIG & $24 \%$ & $37 \%$ & $41 \%$ & $41 \%$ & $39 \%$ \\
$\quad$ high migration & & & & & \\
\hline
\end{tabular}

Source: Authors' computations.

have elevated fertility in the years immediately after migration, while a sizeable proportion of native-born women are still in education and have low fertility by age 25. Thus, the surplus births in the EDU scenario arise because women enrolled in education are not differentiated from women with completed education. This could, however, change in the future if, for instance, future immigrants arrive mainly as students, or are educated at the same or higher levels than native-born women.

Finally, one of the most interesting and relevant results of our analysis with respect to the increasing diversity of European population is the estimated composition of births by the nativity status of the mother. According to our estimates, if recent migration volumes and fertility differentials persist, this share may increase to $28 \%$ by $2055-59$ in the EU28 (Table 5). Naturally, the share is largest (39\% in 2055 59 ) in the high migration scenario - nearly double the share in the EDU scenario that includes only educational attainment differentials. Already in the first projection step, we can see a difference of six percentage points compared to the baseline model (EDU) and the full model (EDU + STUDENT + IMMIG) with differentials by region of birth, duration of stay, and generation.

\section{Conclusions}

Our results illustrate that in the context of educational expansion and increasing population diversity due to immigration, population projections for the EU countries should account for several sources of population heterogeneity. We have shown that how fertility differentials are modelled affects projection results, especially when looking at projected births. Moreover, we have demonstrated that changes in the composition of women of reproductive age in terms of their educational attainment and migrant status can affect both overall TFRs and projected births.

When studying the impact of education on fertility, it is important to capture the postponement effect that is largely driven by educational expansion. This issue 
becomes particularly relevant if we assume that post-secondary education will expand in the future, because this expansion will translate into increasing shares of women staying in education until later reproductive ages. We show that the conventional modelling that does not differentiate between the fertility of students and women with completed education results in higher estimated TFRs. Once we account for the lower fertility rates of women enrolled in higher education, the resulting TFRs decline at current fertility differentials. If education differentials were to narrow, or if the negative relationship between fertility levels and educational attainment were to even reverse, the resulting TFRs and the projected births would also be affected. But in these projections, we do not model these hypothetical situations.

A comparison of the EDU scenario and our full model shows that the TFRs and the projected births are similar under baseline migration assumptions. This holds at the current fertility differentials and at the current composition of immigrant women.

The results for the projected births and TFRs are sensitive to migration assumptions. Our high migration scenario shows steadily increasing numbers of projected births, whereas the scenarios with baseline migration based on recent trends project declining births. The net effect of immigrant fertility on the overall TFR in the EU28 is projected to increase from 0.12 in 2015-2019 to 0.17 in 2055-59 in the scenario with baseline migration, and to 0.25 in $2055-59$ in the scenario with doubled migration. The net effects and the projected increases in births in countries with larger foreign-born population shares are more pronounced under the assumption of sustained high migration.

We have held the composition of immigration flows constant in order to facilitate the assessment of the impact of fertility modelling on the projection results. Naturally, changing the composition of the flows in terms of the countries of origin is another source of heterogeneity that can impact the projection results. The investigation of the impact of such an exercise is beyond the scope of this paper, but it is feasible using experimental what-if scenarios. If more future immigrants come from high-fertility countries, it is possible that the net effect of immigrant fertility will be more pronounced even if the size of the flow does not change. Fertility is expected to decline to close to the replacement level in some of the major sending countries, and this trend could lead to less pronounced differentials between the native-born and immigrants in the future.

The pace of educational expansion in the countries of origin and the selection of migrants may also change in future. In addition, if more female migrants from third countries start immigrating to study or to seek job opportunities rather than for family reasons, we may see immigrant fertility rates converge more quickly to native-bon levels, and narrower fertility differentials between native-born and immigrant women. A hypothetical situation with no differentials between nativeborn and immigrant women with same educational attainment is illustrated by our EDU + STUDENT scenario. 


\section{Acknowledgements}

We are grateful to Patrick Sabourin and Alain Bélanger, who have worked on the development of the CEPAM-mic microsimulation model; and to anonymous reviewers for their valuable comments that helped us to improve the initial draft of this paper.

\section{References}

Afulani, P. A. and J. Asunka 2015. Socialization, adaptation, transnationalism, and the reproductive behavior of sub-Saharan African migrants in France. Population Research and Policy Review 34(4): 561-592. https://doi.org/10.1007/s11113-015-9360-2.

Andersson, G. 2004. Childbearing after migration: Fertility patterns of foreign-born women in Sweden. International Migration Review 38(2): 747-774. https://doi.org/10.1111/j. 1747-7379.2004.tb00216.x.

Barakat, B. and R. E. Durham 2014. World Population and Human Capital in the 21st Century, eds W. Lutz, W. Butz and S. KC, Oxford: Oxford University Press.

Basten, S., T. Sobotka and K. Zeman 2014. Future fertility in low fertility countries. In: World Population and Human Capital in the 21st Century, eds W. Lutz, W. Butz, S. KC, Oxford: Oxford University Press.

Bélanger, A., P. Sabourin, G. Marois, J. Van Hook and S. Vézina 2019. A framework for the prospective analysis of super-diversity. Demographic Research 41: 293-330. https://doi.org/10.4054/DemRes.2019.41.11.

Berrington, A. and S. Pattaro 2014. Educational differences in fertility desires, intentions and behaviour: A life course perspective. Advances in Life Course Research 21: 10-27. https://doi.org/10.1016/j.alcr.2013.12.003.

Blossfeld, H.-P. and J. Huinink 1991. Human capital investments or norms of role transition? How women's schooling and career affect the process of family formation. American Journal of Sociology 97(1): 143-168. https://doi.org/10.1086/229743

Bongaarts, J. 2003. Completing the fertility transition in the developing world: The role of educational differences and fertility preferences. Population Studies 57(3): 321-335. https://doi.org/10.1080/0032472032000137835.

Brzozowska, Zuzanna 2016. Female Education and Fertility under State Socialism in Central and Eastern Europe. Population 70(4): 689-725. https://doi.org/10.3917/popu.1504.0731.

Caselli, G., S. Drefahl, C. Wegner-Siegmundt and M. Luy 2014. Future mortality in low mortality countries. Chapter 5 in World Population $\mathcal{E}$ Human Capital in the Twenty-First Century, eds W. Lutz, et al., 226-272. Oxford University Press.

Castro Martin, T. and L. Rosero-Bixby 2011. Motherhood and transnational borders: Immigrants' women fertility in Spain. Revista Internacional De Sociologia 69: 105-137. https://doi.org/10.3989/ris.2011.iM1.388.

Colby, S. L. and J. M. Ortman 2015. Projections of the Size and Composition of the U.S. Population: 2014 to 2060. Population estimates and projections. U.S. census Bureau: U.S. Department of Commerce. https://www.census.gov/content/dam/Census/library/ publications/2015/demo/p25-1143.pdf [Last accessed 28.3.2019]. 
Coleman, D. 2006. Immigration and ethnic change in low-fertility countries: A third demographic transition. Population and Development Review 32(3): 401-446. https: //doi.org/10.1111/j.1728-4457.2006.00131.x.

Dubuc, S. 2012. Immigration to the UK from high-fertility countries: Intergenerational adaptation and fertility convergence. Population and Development Review 38(2): 353-368. https://doi.org/10.1111/j.1728-4457.2012.00496.x.

Dubuc, S. 2017. Fertility and education among British Asian women: A success story of social mobility? Vienna Yearbook of Population Research 15: 269-291. https://doi.org/10. 1553/populationyearbook2017s269.

Goujon A., V. Skirbekk, K. Fliegenschnee and P. Strzelecki 2007. New times, old beliefs: Projecting the future size of religions in Austria. Vienna Yearbook of Population Research 5: 237-270. https://doi.org/10.1553/populationyearbook2007s237.

Hertz, T., T. Jayasundera, P. Piraino, S. Selcuk, N. Smith and A. Verashchagina 2008. The Inheritance of Educational Inequality: International Comparisons and Fifty-Year Trends. The B.E. Journal of Economic Analysis E Policy 7(2). https://doi.org/10.2202/1935-1682. 1775 .

Jalovaara, M., G. Neyer, G. Andersson, J. Dahlberg, L. Dommermuth, P. Fallesen and T. Lapegard 2019. Education, gender, and cohort fertility in the Nordic countries. European Journal of Population 35: 563-586. https://doi.org/10.1007/s10680-018-9492-2.

Jeejebhoy, S. 1995. Women's education, autonomy and reproductive behavior: Experience from developing countries. Oxford: Clarendon Press.

Krapf S. and K. Wolf 2016. Persisting differences or adaptation to German fertility patterns? First and second birth behavior of the 1.5 and second generation Turkish migrants in Germany. In: Social Demography Forschung an der Schnittstelle von Soziologie und Demografie, eds K. Hank and M. Kreyenfeld. Kölner Zeitschrift für Soziologie und Sozialpsychologie (Sonderheft 55/2015). Wiesbaden: Springer. https://doi.org/10.1007/ 978-3-658-11490-9_7.

Kravdal, Ø. 2002. Education and fertility in sub-Saharan Africa: Individual and community effects. Demography 39(2): 233-250. https://doi.org/10.1353/dem.2002.0017.

Kravdal, $\varnothing$. and R. R. Rindfuss 2008. Changing relationships between education and fertility: A study of women and men born 1940 to 1964. American Sociological Review 73(5): 854-873. https://doi.org/10.1177/000312240807300508.

Kulu, H. 2005. Migration and fertility: Competing hypotheses re-examined. European Journal of Population 21(1): 51-87. https://doi.org/10.1007/s10680-005-3581-8.

Kulu, H. and N. Milewski 2007. Family change and migration in the life course: An introduction. Demographic Research 17: 567-590. https://doi.org/10.4054/DemRes.2007. 17.19.

Kulu, H., T. Hannemann, A. Pailhe, K. Neels, S. Krapf, A. Gonzalez-Ferrer and G. Andersson 2017. Fertility by birth order among the descendants of immigrants in selected European countries. Population and Development Review 43(1): 31-60. https://doi.org/10.1111/ padr.12037.

Kulu, H., N. Milewski, T. Hannemann and J. Mikolai 2019. A decade of life-course research on fertility of immigrants and their descendants in Europe. Demographic Research 40(46): 1345-1374. https://doi.org/10.4054/DemRes.2019.40.46. 
Lutz, W. and A. Goujon 2001. The world's changing human capital stock: Multi-state population projections by educational attainment. Population and Development Review 27(2): 323-339. https://doi.org/10.1111/j.1728-4457.2001.00323.x.

Lutz, W. and V. Skirbekk 2014. How education drives demography and knowledge informs projections. World population and human capital in the 21 st century, eds W. Lutz, W. Butz, S. KC. Oxford: Oxford University Press.

Lutz, W., W. Butz and S. KC (eds) 2014. World population and human capital in the 21st century. Oxford: Oxford University Press.

Lutz, W., A. Goujon, S. KC, M. Stonawski and N. Stilianakis (eds) 2018. Demographic and human capital scenarios for the 21st century: 2018 assessment for 201 countries. Luxembourg: Publications Office of the European Union.

Marois, G., P. Sabourin and A. Bélanger 2019. Forecasting human capital of EU member countries accounting for sociocultural determinants. Journal of Demographic Economics 85(3): 231-269. https://doi.org/10.1017/dem.2019.4.

Mortimer, J. T., S. Oesterle and H. Krueger 2005. Age norms, institutional structures, and the timing of markers of transition to adulthood. Advances in Life Course Research 9: 175-203. https://doi.org/10.1016/S1040-2608(04)09007-0.

Mussino, E. and S. Strozza 2012. The fertility of immigrants after arrival: The Italian case. Demographic Research 26(4): 99-130. https://doi.org/10.4054/DemRes.2012.26.4.

Neels, K., M. Murphy, M. Ní Bhrolcháin and É. Beaujouan 2017. Rising educational participation and the trend to later childbearing. Population and Development Review 43(4): 667-693. https://doi.org/10.1111/padr.12112.

Ní Bhrolcháin, M. and É. Beaujouan 2012. Fertility postponement is largely due to rising educational enrolment. Population Studies 66(3): 311-327. https://doi.org/10.1080/ 00324728.2012 .697569$.

Persson, L. and J. Hoem 2014. Immigrant fertility in Sweden, 2000-2011: A descriptive note. Demographic Research 30(30): 887-898. https://doi.org/10.4054/DemRes.2014.30.30.

Raymer, J., Y. Shi, J. O'Donnel and N. Biddle 2018. Multistate projections of Australia's indigenous population: Interacting area group and identification status change. Vienna Yearbook of Population Research 16: 135-162. https://doi.org/10.1553/ populationyearbook2018s135.

Raymer, J., A. Wiśniowski, J. J. Forster, P. Smith and J. Bijak 2013. Integrated modeling of european migration. Journal of the American Statistical Association 108(503): 801-819. https://doi.org/10.1080/01621459.2013.789435.

Rees, P. 2002. New models for projecting UK ethnic group populations at national and subnational scales. In: Population Projections by Ethnic Group: A Feasibility Study, ed. Haskey, J., 27-51. London: Stationery Office.

Rindfuss, R. R. and L. Bumpass 1980. Education and fertility: Implications for the roles women occupy. American Sociological Review 45(3): 431-447.

Robarts, J., A. Berrington and T. McGowan (eds) 2015. The fertility of recent migrants to England and Wales: interrelationships between migration and birth timing (ESRC Centre for Population Change Working Papers, 65). Southampton: University of Southampton. https://eprints.soton.ac.uk/377909/ [Last accessed 28.3.2019] 
Rogers, A. 1980. Introduction to multistate mathematical demography. Environment and Planning A: Economy and Space 12(5): 489-498.

Rogers, A. 1986. Parameterized multistate population dynamics and projections. Journal of American Statistical Association 81(393): 48-61, https://doi.org/10.1080/01621459.1986. 10478237.

Rogers, A. and L. J. Castro 1981. Model Migration Schedules. IIASA Research Report. IIASA, Laxenburg, Austria: RR-81-030

Rogers, A., J. Little and J. Raymer 1999. Disaggregating the historical sources of regional foreign-born and native-born population growth in the United States: A new method with applications. International Journal of Population Geography 5: 449-475.

Roig Vila, M. and T. Castro-Martín 2007. Childbearing Patterns of Foreign Women in a New Immigration Country: The Case of Spain. Population 62(3): 351-379. https://doi.org/10.3917/popu.703.0419.

Sabourin, P., G. Marois and A. Bélanger 2017. The Base Population of the CEPAM Microsimulation Model, Technical Report - Version 1.0, International Institute for Applied Systems Analysis, $30 \mathrm{p}$.

Schoen, R. 1988. Modeling Multigroup Populations. Plenum Press: New York.

Shavit, Y. and H.-P. Blossfeld. 1993. Persistent inequality: Changing educational attainment in thirteen countries. Social Inequality Series. Boulder: Westview Press.

Sobotka, T. 2008. Overview chapter 7: The rising importance of migrants for childbearing in Europe. Demographic Research 19(9): 225-248. https://doi.org/10.4054/DemRes.2008. 19.9.

Stonawski, M., V. Skirbekk, C. Hackett, M. Potančoková, P. Connor and B. Grim 2015. Global population projections by religions: 2010-2050. In: Yearbook of International Religious Demography, 101-129. Brill. https://doi.org/10.1163/9789004297395_004.

Toulemon, L. 2004. Fertility among immigrant women: New data, a new approach. Population and Societies n. 400. Paris: INED. https://www.ined.fr/fichier/s_rubrique/18833/publi_ pdf2_pop_and_soc_english_400.en.pdf [Last accessed 28.3.2019].

van Bavel, J. 2014. The mid-twentieth century baby boom and the changing educational gradient in Belgian cohort fertility. Demographic Research 30(33): 925-962. https://doi. org/10.4054/DemRes.2014.30.33.

van Imhoff, E. and W. Post 1998. Microsimulation Methods for Population Projections. Population: An English Selection 10: 97-138.

Wood, J., K. Neels and T. Kil 2014. The educational gradient of childlessness and cohort parity progression in 14 low fertility countries. Demographic Research 31(46): 1365-1416. https://doi.org/10.4054/DemRes.2014.31.46.

Open Access This article is published under the terms of the Creative Commons Attribution 4.0 International License (https://creativecommons.org/licenses/by/4.0/) that allows the sharing, use and adaptation in any medium, provided that the user gives appropriate credit, provides a link to the license, and indicates if changes were made. 